\title{
El marco informativo de la iniciativa estatal de palestina en los medios colombianos
}

\section{The informative frame of the Palestine state initiative in the Colombian media}

\author{
Jairo Dario Velásquez Espinosa (M.A.) ${ }^{1}$ \\ Universidad de La Sabana - Colombia \\ Jairo.velasquez@unisabana.edu.co
}

Recibido: 30 de agosto de 2012 Aceptado: 23 de septiembre de 2012

\section{Resumen}

Colombia, por su lugar temporal en el Consejo de Seguridad de la Organización de Naciones Unidas durante el 2011, se vio convertida en protagonista del ciclo noticioso creado por la campaña mundial en pro del reconocimiento de Palestina como estado miembro de la Asamblea General de la organización internacional mencionada. Esta investigación estudió, cuantitativa y cualitativamente, el tratamiento de la información publicada sobre este hecho en cuatro diarios colombianos (El Tiempo, El Espectador, El País y El Heraldo), dos nacionales y dos regionales, para que sobre ella se pudiera analizar el marco (frame) de la realidad construido desde el cubrimiento noticioso. El análisis se centró en las publicaciones hechas entre julio y diciembre de 2011 para poder tener una perspectiva completa del proceso antes, durante y después de la presentación de la propuesta ante la Asamblea. La metodología utilizada en el desarrollo de la pesquisa se centró en los postulados defendidos desde la teoría del framing, en especial con las categorías de medición planteadas por Taknard (2001). La exploración mostró que la imagen que sobre temas internacionales se construye en los medios colombianos es pobre y, en su mayoría, basada en la visión que de los problemas tienen terceros representados en las diferentes Agencias de Noticia utilizadas. Además, se pudo comprobar que los temas externos tuvieron más cobertura en los periódicos de tirada nacional, que asumieron posiciones editoriales acordes con las planteadas por el Gobierno colombiano, referenciando apenas la postura de los palestinos y casi que apoyando el rechazo a su propuesta.

\footnotetext{
${ }^{1}$ Investigación realizada con los estudiantes del programa de Comunicación Social y Periodismo, Juan F. Bernal M. y Laura Benedetti.
} 


\section{Abstract}

Colombia, due to its temporary seat on the UN Security Council in 2011, became a main player in the news cycle prompted by the Palestinian world campaign for recognition as a member state to the General Assembly. This research analyzed, from a quantitative and qualitative perspective, the treatment of the information on this topic by four Colombian daily newspapers (El Tiempo, El Espectador, El País and El Heraldo), two of which have national, and the other two regional, distribution. They were studied to understand the framing of reality created in the news coverage. The analysis focused on the published material between July and December 2011 to have the whole perspective of the process from before, during, and after, the proposal was sent to the General Assembly. The methodology used in this research is based on the central tenets of the framing theory, specially the units of measure designed by Taknard (2001). The review showed that the image of international affairs in Colombian printed media is poor, and mostly based on the perspective of third parties - such as the News Agencies cited -. Furthermore, it was found that foreign topics had more coverage in nation-wide newspapers, which took stances close to the ones adopted by the government, barely mentioning the Palestinian perspective and almost supporting the rejection of the proposal.

Palabras Clave: cubrimiento; Palestina; Colombia; framing; El Tiempo; El Espectador; El Heraldo; El País.

Key Words: Cover; Palestine; Colombia; framing, El Tiempo; El Espectador; El Heraldo; El País.

\section{Introducción}

En el 2011, bajo la sombra de la Primavera Árabe, el gobierno de la Autoridad Nacional Palestina, encabezado por Mahmoud Abbas, revivió el postergado tema del reconocimiento internacional del Estado de Palestina y llevó la propuesta al seno de la Asamblea General de las Naciones Unidas. Un hecho enmarcado en un longevo conflicto palestino israelí que sigue produciendo continuos enfrentamientos entre las diferentes potencias de la sociedad mundial.

Frente a esta coyuntura, el interés de los medios de comunicación fue grande y aprovechado por los gobiernos que apoyaban o rechazaban la iniciativa. Cada reacción de estos actores tuvo impacto en la recepción y la emisión de la información por parte de las empresas mediáticas, generándose dos agendas temáticas paralelas que dejaban ver a la clara y profunda división que ha construido sobre el asunto se en las últimas seis décadas. 
Ese interés mediático es precisamente lo que motivó esta investigación. El artículo presentará un análisis del cubrimiento que se hizo de la propuesta de Palestina en la sección internacional, entre julio y diciembre de 2011, de cuatro diarios colombianos: dos nacionales y dos regionales: El Espectador, El Tiempo, El Heraldo (Barranquilla) y El País (Cali) respectivamente.

La observación se realizó siguiendo los postulados de la teoría del framing para el análisis cuantitativo y cualitativo de la información, identificando los marcos sobre los cuales se construyeron las noticias seleccionadas.

\subsection{Marco conceptual}

\subsubsection{El anhelo de ser un Estado}

El 23 de septiembre de 2011, Palestina llevó a la Organización de Naciones Unidas una propuesta con la que buscaba ser aceptada como Estado, o al menos como un Estado observador con una silla en el organismo internacional. Mahmoud Abbas, presidente de la Autoridad Nacional Palestina (ANP), presentó la petición formal al secretario general de la Organización Ban Ki-moon, con el ánimo de que se discutiera tanto en la Asamblea General del organismo como en la reunión posterior del Consejo de Seguridad en octubre. Sin embargo, el deseo de los palestinos, y de varios países en el mundo que apoyaron su causa, fue rechazado tras una extensa campaña de promoción y convencimiento.

La posibilidad de convertirse en un Estado se vio truncada, una vez más, por la negativa de Israel de avanzar en un proceso de paz bilateral, que al final impulsaría el veto de los Estados Unidos en el Consejo de Seguridad de la ONU, al argumentar que sin este acuerdo entre los dos actores era inconveniente avanzar en la causa del reconocimiento palestino (Barreñada, 2011: 3)

Este ha sido un problema que ha viajado por generaciones, que con el inicio del siglo XX se vio intensificado por la llegada de los británicos al terminar la Primera Guerra Mundial y su posterior promesa de solucionar el problema judío y el problema musulmán en la región.

Con el fin de la Segunda Guerra Mundial, y siguiendo los principios determinados en la Carta del Atlántico en 1941 y en la Carta de las Naciones Unidas en 1945, la cuestión Palestina tuvo un nuevo impulso cuando los británicos, deprimidos y golpeados por la dureza del conflicto, elevaron a la recién creada Organización de las Naciones Unidas, la propuesta buscar soluciones al problema y fue creada una comisión que recomendaría que el territorio fuera dividido en un Estado árabe y un Estado judío con un estatuto internacional especial para la ciudad de Jerusalén, bajo la autoridad administrativa de la ONU (DIPNU, 2003: 5). 
La oportunidad de obtener concepto positivo para palestinos e israelíes se presentó en noviembre de 1947 donde, como lo plantea Spizzamiglio (2005: 3), "la Asamblea General intentó dar solución al conflicto mediante el establecimiento de la resolución 181, la cual instauraba la partición del territorio en dos estados (...) sin embargo, ésta fue desoída", pues en aquella reunión solo se tuvo en cuenta la creación de un Estado israelí que contó con 33 votos a favor. ${ }^{2}$

Según Brunetto (2011: 12), desde un comienzo "los árabes reclamaban que Palestina fuera un Estado unitario con mayoría árabe permanente y que alcanzara la independencia como tal". Este punto, considerado como el de mayor importancia, no se cumplió, sino que por el contrario, el 29 de noviembre de 1947 se tomaron varias decisiones en las que se exigió la terminación del mandato británico, la retirada progresiva del ejército y la fijación de fronteras entre los dos Estados y Jerusalén" (DIPNU, 2003: 10).

La admisión de Israel como un Estado independiente a las Naciones Unidas fue el detonante de los conflictos territoriales, políticos e incluso culturales que han mantenido dividida la región hasta ahora. Un enfrentamiento que inició con la Guerra de la Independencia (15 de mayo de 1948 al 6 de enero de 1949) (Martínez, 1999: 358), que trajo consigo el inicio del problema de los cientos de refugiados palestinos que huyeron a otros territorios. En $1956 \mathrm{el}$ conflicto se escalaría con la crisis del Canal del Suez y la segunda guerra árabe-israelí, entre Egipto e Israel (Martínez, 1999: 358).

En este punto, los palestinos empezaron a intentar construir una estructura política que representara sus intereses, por lo que en 1964, con el apoyo de los Estados Árabes, crean la Organización para la Liberación de Palestina (OLP), que se situó como legítima representante del pueblo (Caruana, 2002:13).

Para 1967, y con la excusa de aumentar su control en la zona y así protegerse de las amenazas que lo rodeaban, Israel lanza un ataque fulminante contra Egipto, Jordania y Siria, ocupando el Sinaí, la Franja de Gaza, la Ribera Occidental, incluida Jerusalén oriental, y los Altos del Golán, en su parte siria (DIPNU, 2003: 19), territorios que aun hoy, en su mayoría, están en el centro de la disputa internacional sobre el futuro de Palestina.

En este mismo año la ONU, por medio del Consejo de Seguridad, aprobó la resolución 242, en la que sentaba los principios para lograr una solución pacífica en el Medio Oriente (DIPNU, 2003: 19), desconociendo el plan inicial de partición de 1947 para incorporar las

\footnotetext{
${ }^{2}$ Las naciones que votaron a favor fueron: Australia, Bélgica, Bolivia, Brasil, Canadá, Costa Rica, Checoslovaquia, Dinamarca, República Dominicana, Ecuador, Estados Unidos, Francia, Guatemala, Haití, Islandia, Liberia, Luxemburgo, Holanda, Nueva Zelanda, Nicaragua, Noruega, Panamá, Paraguay, Perú, Filipinas, Polonia,Suecia, Sudáfrica, Unión Soviética, Uruguay, Venezuela; 13 en contra: Afganistán, Cuba, Egipto, Grecia, India, Irán, Iraq, Líbano, Pakistán, Arabia Saudí, Siria, Turquía, Yemen, y 10 Estados se abstuvieron: Argentina, Chile, China, Colombia, Salvador, Etiopía, Honduras, México, Reino Unido y Yugoslavia (Söderblom, 2003: 10).
} 
nuevas fronteras israelitas. Posición que el gobierno del presidente de los Estados Unidos, Barack Obama, defiende como la mejor salida al conflicto actual.

Para la década de los 70, el conflicto se encontraría, en el 73, con el ataque de Egipto y Siria a Israel y siendo repelidos por este, añadiendo aun más zonas a su territorio (Martínez, 1999: 359); y en el 74, la ONU otorgó a la OLP el estatuto de observador y la Conferencia Árabe la reconoció como representante legítima del pueblo Palestino (Caruana, 2002: 14). En ese entonces Yasser Arafat, cabeza de la OLP, comenzó a buscar consenso internacional en medio de acciones armadas ilegales contra bandos israelitas, lo que llevó a Israel a catalogarla como una organización terrorista y así minimizar el liderazgo y la capacidad de negociación de los palestinos (Daher, 2002: 6).

En 1987 los palestinos decidieron levantarse contra los ataques constantes de Israel a través del movimiento popular palestino conocido como 'Intifada', que se originó el "9 de diciembre de 1987 y que canalizó la frustración acumulada de miles de palestinos tras años de ocupación" (Urrutia, 2011: 9). Esta reacción no pudo ser contenida por Israel dado que los focos de ataque se centraron en varias zonas de la frontera.

Un año después, en 1988, se produjo "la declaración de independencia del Estado Palestino (...) logrando el reconocimiento inmediato por más de 105 países" (Caruana, 2002: 17, 18), suceso que sentó precedente para la OLP aunque sin el apoyo de la ONU. Es por esto que Barreñada (2011: 2) afirma que "desde un punto de vista formal, el Estado de Palestina existe desde 1988 y la declaración de Argel [ese año] fue el ejercicio del derecho de autodeterminación".

En 1993, con Rusia y Estados Unidos como testigos, los actores del conflicto firmaron la Declaración de Principios sobre las disposiciones relacionadas con un Gobierno Autónomo Provisional en donde las partes acordaron, poner fin a decenios de enfrentamientos y conflictos, reconocer sus legítimos derechos políticos mutuos, tratar de vivir en un régimen de coexistencia pacífica y de dignidad y seguridad mutuas, llegar a una solución de paz justa, duradera y global y, a una reconciliación histórica por conducto de un proceso político convenido (AS, 1993).

Este mismo año tanto Palestina como otras regiones árabes intentaron sumirse en un proceso de paz con Israel cuyo fin era terminar el conflicto por el cual pasaban: "la consecuencia más tangible de esta política de paz israelí fueron los acuerdos de Oslo entre israelíes y palestinos, que ofreció un marco de común acuerdo para una solución al conflicto" (Martínez, 1999: 361).

Sin embargo, para 1995, las negociaciones se truncaron con el asesinato del entonces primer ministro israelí, Yitzahk Rabin. El acuerdo de paz se postergó hasta el año 2000, cuando el entonces presidente de Estados Unidos, Bill Clinton, "invitó a los dirigentes de 
Israel y de la Autoridad Palestina a mantener conversaciones de paz en la residencia presidencial de Camp David" (DIPNU, 2003: 59). Estos diálogos no tuvieron fuerza debido a que ese mismo año se dio inició a la segunda Intifada (2000 - 2005) donde "al menos 50 personas murieron y unas 1.500 sufrieron heridas, la mayoría palestinas" (DIPNU, 2003: 60).

Ya en el Siglo XXI, específicamente en el 2001, Ariel Sharon, el nuevo primer ministro israelí, emprendió una ofensiva contra Palestina favorecido por las nuevas doctrinas del Presidente George W. Bush en su guerra contra el terrorismo y el eje del mal (Daher, 2002: 11). Los ataques contra la población palestina se hicieron cada vez más constantes, se impulsaron las construcciones de nuevos asentamientos internacionales en las zonas ocupadas, haciendo cada vez más lejana cualquier ilusión por un acuerdo de paz.

En 2011, el gobierno palestino, presidido por Mahmoud Abbas, decidió buscar la creación de un Estado palestino que pudiera defender y reclamar sus territorios teniendo en cuenta el largo proceso histórico que implicó defenderse de los israelíes. Posterior al anuncio de la propuesta palestina, se generó una campaña mundial para buscar el apoyo de terceros con el fin de llegar a la Asamblea General habiendo conseguido la aprobación de la mayoría de los Estados miembros. Una propuesta que, a pesar de los 117 países que ya la reconocían, fue votada negativamente por Israel, Estados Unidos e Inglaterra, además de las abstenciones o ausencias de Alemania, Italia, algunos del este de Europa, Canadá y Colombia; terminando así el sueño del reconocimiento político de Palestina.

Finalmente, de acuerdo con Urrutia (2011: 42), la idea de proclamar un Estado aparece como una ilusión que no conducirá a la autodeterminación efectiva del pueblo palestino, ya que por sí sola no equivale a una soberanía efectiva ni a la independencia. Se considera que optar por esta vía deja todavía sin respuesta algunas de las aspiraciones más relevantes del pueblo palestino, por ejemplo, las relacionadas con la capitalidad de Jerusalén o el destino de los refugiados.

\subsubsection{Marco Teórico: Teoría de encuadre o framing}

La investigación trabaja sobre el análisis de los encuadres en los que se presenta la información sobre un proceso político específico, como lo es en este caso el reconocimiento internacional de un nuevo actor estatal.

Para este fin se dejó de lado la idea clásica de la objetividad periodística y se abrazó la idea de que los hechos siempre se cuentan desde un enfoque particular, determinado ya sea por motivos personales y profesionales, tal como lo defiende la teoría del Framing (Sadaba, 2004).

Es una teoría que permite estudiar el entorno y las herramientas con las que los periodistas pueden conectarse con la sociedad, contribuyendo al buen o al mal entendimiento de otros países y culturas, permitiendo la creación de nuevas definiciones de la realidad que al final se pone a disposición del público. 
En sus orígenes, el concepto de Framing se encuentra desde enfoques sociológicos y psicológicos que buscan interpretar los modos de conocimiento humano y no desde los estudios de comunicación de masas. Este solo se trasladó al estudio de los medios de comunicación, cuando sus efectos se empezaron a vincular con cuestiones de tipo cognitivo, como sucede con los trabajos de Agenda-Setting, con la que frecuentemente se vincula al Framing (Sádaba, 2004: 70).

La teoría del "encuadre" se ubica en el polo opuesto de las teorías objetivistas que presentaban a los medios de comunicación como simples espejos de la realidad: antes de dar cualquier información, los periodistas hacen una selección de atributos que son los que finalmente entregan al público, narrando los acontecimientos con un enfoque particular. Cómo es lógico, dentro de ese enfoque se encuentran las influencias personales y profesionales con las que el periodista elabora los mensajes, que hacen que los acontecimientos adquieran marcos o frames (Sádaba, 2006: 1).

Los enfoques de la información se consideran ahora una parte esencial y legítima de los modos de comunicar. El encuadre que recibe la información, lejos de resultar una limitación o incluso de parecer inadmisible para el trabajo periodístico, resulta de un valor político, social y cultural indiscutible y un componente genuino de la profesión (Sádaba, 2004: 71). El periodismo puede tornarse incomprensible sin tener en cuenta los enfoques, que son claves para analizar el mensaje contenido en los medios.

Tuchman plantea que la creación de significados por parte de los comunicadores está inmersa en procesos institucionales propios de cada medio de comunicación (Tuchman, 1978). Por eso, desde la teoría del Framing, se considera a los medios como agentes que conceptualizan la realidad bajo unos parámetros determinados y no como canales que simplemente la estructuran para transmitirla de modo neutral.

En el 2001, James W. Taknard se preocupará por hacer medible el Framing, de manera cuantitativa, de modo que sea visible la forma como los encuadres pueden influenciar el proceso de creación de las noticias. Dice que el encuadre es la idea organizadora central del contenido informativo que entrega un contexto y sugiere el tema desde la selección, el énfasis, la exclusión y la elaboración.

Taknard estableció (2001: 98) variables para explicar el proceso del Framing: la primera es utilizando el símil del marco de una fotografía para afirmar que en la construcción informativa aísla cierto material y centra la atención sobre un objeto que ya ha sido sometido a un proceso de selección, énfasis y exclusión. Ese mismo marco suministra un tono que permite conocer el contexto que llevará a la interpretación. Por último, nos encontramos con el símil del marco arquitectónico, que en el caso de la comunicación nos hablará del marco que organizará la historia y luego impulsará su construcción. 
Ahora bien, teniendo en cuenta el objeto de estudio de esta investigación, conviene aclarar que, aunque en todas las temáticas se pueden percibir los efectos del Framing, estos se acentúan más en las noticias internacionales donde el poder mediático para imponer una determinada visión de la realidad es prácticamente absoluto, ya que los temas tratados son en su mayoría desconocidos y no se pueden experimentar directamente.

\subsubsection{Estado del Arte}

El conflicto entre Palestina e Israel ha sido materia constante de estudio en diferentes campos del conocimiento, especialmente en el de las ciencias humanas. Desde un punto de vista periodístico, las investigaciones han servido para mostrar y evidenciar la violencia entre las partes, así como los intentos de paz propuestos por otros gobiernos y organismos internacionales. Partiendo de este hecho, los estudios sobre la labor informativa que han cumplido los medios de comunicación han sido muy pocos.

Las investigaciones enfocadas en el periodismo internacional sobre este conflicto se centran principalmente en la visión y el manejo que los medios europeos le han dado. Sin embargo, en lo que se refiere a América Latina, específicamente desde las empresas informativas de Colombia, no existen estudios profundos sobre la percepción mediática y popular respecto a los sucesos que están ocurriendo desde hace más de cinco décadas en esta zona de Oriente Medio y que hoy en día son noticia tanto en los medios tradicionales como en la web.

En cuanto a las investigaciones que se desarrollan en torno a la comunicación digital y su rol en el conflicto, se orientan a la utilización de las redes sociales (digitales) tal como ocurrió con el inicio de la denominada Primavera Árabe. La comunicación digital se ha convertido en un campo indispensable para el desarrollo de las noticias y el traspaso de la información por cualquier medio de comunicación masiva.

Actualmente los consumidores de noticias tienen la posibilidad de informarse en los medios de comunicación internacionales, que en casos como la disputa por la Franja de Gaza, se convierten en una fuente más confiable y cercana a los hechos. Una oportunidad que no aprovechan aquellos de corte más nacional, o local, que siguen dejando de lado la profundidad en el manejo de las informaciones internacionales.

Al fijarnos exclusivamente en el conflicto de los países árabes, notamos que las investigaciones periodísticas con respecto a este hecho se enfocan, en su gran mayoría, en la participación de los ciudadanos mediante las distintas redes sociales El portal web de la agencia Europe Press hace hincapié en un estudio realizado por el Centro Internacional de Asistencia a los Medios de comunicación (CIMA) donde afirma que "las redes sociales han superado ya a los periódicos como medio de información en los países árabes".

Ahora, si en algo concuerdan las investigaciones periodísticas realizadas en torno al conflicto palestino-israelí es que, no solo su presentación en los medios de comunicación ha variado 
mucho según el país de origen del medio y la línea editorial del mismo, sino también en la percepción sesgada y desenfocada que tienen los países occidentales con referencia a los de oriente.

En este escrito se toman como muestra de comparación la tesis doctoral La línea editorial de ABC, El País, El Mundo y La Vanguardia frente al conflicto Palestino-Israelí de la Doctora Ana María Córdoba (2009) y La construcción discursiva del evento conflictivo en las noticias por televisión, tesis de postgrado de Nora Kaplan (2009).

En principio, la disputa de la Franja de Gaza importaba tan solo a los afectados en primer grado. Con el tiempo, comenzó a crear eco internacional debido al fenómeno de la globalización, ${ }^{3}$ algo que, con la propuesta que llevó Palestina ante la ONU, permite comprobarse por la amplitud en el cubrimiento informativo, desde diferentes perspectivas, hecho por los medios, incluso teniendo en cuenta la posición frente al asunto que hubiera asumido su país de origen.

"La prensa convierte los hechos en noticias y los sucesos en acontecimientos, ejerciendo un filtro entre lo real y la realidad, entre lo inteligible per se, y lo susceptible de ser explicado o comprendido" (Sánchez, 1998 citado en Córdoba, 2009: 18). Pero, también crea nuevos canales nacionales e internacionales por lo que la publicación de las noticias sobre el extranjero, en este caso sobre los conflictos en oriente medio, convierte al periodista en el narrador de la historia.

Por último, cada uno de los artículos analizados fue creado por periodistas que se encargan de la investigación y redacción del tema mismo; sin embargo, a los ojos de la audiencia, la publicación es presentada por un ente único con una postura única. Así, aunque el autor adopte una postura objetiva y determinada, esta está sujeta a la línea editorial del medio para el que trabaja.

Teóricamente esta es encuadrada dos veces y manipulada. ${ }^{4}$ Es decir, si el medio de comunicación tiene como política que las noticias deben alejarse del sensacionalismo, donde los hechos se presentan de manera sugestiva, es deber del periodista adecuar la noticia respetando esos límites.

Entonces Existe lo que Kaplan (2009: 337) denomina despersonalización que "coincide con un total distanciamiento en la relación con el espectador" donde el autor de la noticia no se muestra. Y esa brecha además está aún más mediada teniendo en cuenta que la información es producida por terceros, como agencias de noticias internacionales o los medios locales y

\footnotetext{
${ }^{3}$ De acuerdo con Anthony Giddens (1990: 65), la globalización puede ser comprendida como un proceso histórico que ha implicado un profundo cambio o transformación en la organización social, abriendo la posibilidad de vincular comunidades distantes y expandiendo el alcance del poder de las relaciones a través de regiones y continentes.

${ }^{4}$ Esta manipulación hace referencia al manejo indirecto e involuntario de la percepción que puede dársele a una noticia que se atiene a lo fáctico.
} 
nacionales de la zona donde se está dando el suceso. Son pocas las veces en las que se envían corresponsales a cubrir los conflictos y más cuando son en territorios lejanos.

Es de recordar que cuando una noticia versa sobre un suceso internacional, la mayoría de las veces, los receptores son extraños al caso de origen razón por lo que la información debe entregarse en un formato simple. El uso de figuras, afirmaciones implícitas, citas textuales, paralelismos, comillas, signos de puntuación y otras cuestiones de retórica y redacción logran dar o restar poder al significado de las palabras mismas (Córdoba, 2009: 287).

\section{Metodología}

\subsection{El manejo de las noticias internacionales en Colombia}

Los diarios utilizados como muestra para este ejercicio se han caracterizado por ser constantes en la publicación de noticias y por abarcar contenidos de corte internacional.

Se seleccionaron las versiones Web de El Tiempo de Bogotá, El Espectador de Bogotá, El País de Cali y El Heraldo de Barranquilla, entre julio a diciembre de 2011, periodo que abarca los momentos previos, el desarrollo y las consecuencias de la Asamblea General de Naciones Unidas donde la Autoridad Palestina presentaría su propuesta.

Algunos de los criterios de selección fueron: ser diarios escritos con una plataforma Web, estar entre los de mayor tiraje nacional, no se gratuitos, que su principal idioma fuera español y que su archivo fuese de acceso gratuito.

Se optó por desarrollar un recuento sistemático que permita mostrar cómo los medios de comunicación presentaron las noticias y sí durante el periodo seleccionado se evidenció alguna postura determinada sobre el conflicto palestino-israelí.

Se analizó el marco creado por los emisores de los mensajes (medios de comunicación) para luego inferir la imagen de la realidad que se le estaba entregando a la opinión, desde el análisis de una matriz construida siguiendo los postulados de Broullón, Hernández, López y Pereira (2005).

En esa matriz se consagraría la información desde diferentes categorías, todas defendidas por Taknard (2001), que permitirían luego hacer el análisis cuantitativo de la información. Se ha pensado que la matriz cuente con variables cuantitativas como:

- Cantidad de noticias: Confrontar cuáles tienen mayor incidencia según el número de noticias publicadas por cada medio de comunicación.

- Variación de fechas: Comparar las fechas en que se publicaron las noticias en cada medio, teniendo en cuenta la importancia de las publicadas en el mes de septiembre 
(mes en que se llevó a cabo la Asamblea de la ONU) y aquellas fechas que se repitieron.

- Protagonistas de la noticia: número de veces que se mencionan diferentes actores dentro de las noticias.

- Escenario: en qué sitio geográfico se desarrolla la noticia.

- Temas: cuál es la orientación que los medios de comunicación le dan a la noticia teniendo en cuenta la coyuntura.

- Procedencia: número de veces en que se repitió una fuente.

- Número de palabras (por noticia): importancia de la noticias teniendo en cuenta la cantidad de palabras (relacionándolas con la fecha, con el objetivo de conocer la relevancia que tuvieron en momentos clave).

En cuanto al análisis cualitativo se busca una aproximación a las percepciones y actitudes sociales emergentes de la lectura y el análisis de las noticias gracias a categorías como:

- Detalles externos: aspectos que se mencionaron en las noticias pero que nada tenían que ver con el tema principal de la propuesta presentada por Palestina.

- Cantidad de frases en negrita y frase más destacada: evidenciar la orientación que los medios le dan a la noticia teniendo en cuenta los textos resaltados.

Los parámetros para la obtención de cada una de las variables del análisis, se siguieron las siguientes temáticas claves frente al tema seleccionado:

- Revisión de noticias de acuerdo con el tema planteado de la propuesta Palestina de aceptación como Estado ante la ONU

- Revisión de noticias cronológicamente en un periodo de seis meses que comprende desde el 1 ro de julio de 2011 y hasta el 31 de diciembre de 2011

- Revisión de noticias en el archivo web de cada uno de los medios

- Lectura y seguimiento de cada una de las variables para el análisis

Al final, el formato diseñado para desarrollar investigación y extraer los datos de acuerdo con las variables propuestas es el siguiente:

\begin{tabular}{|l|l|l|l|l|l|l|l|l|l|}
\hline $\begin{array}{l}\text { Número } \\
\text { de } \\
\text { noticia }\end{array}$ & Fecha & Medio & Titular & $\begin{array}{l}\text { Número } \\
\text { de } \\
\text { palabras }\end{array}$ & Protagonistas & Escenario & Tema & Procedencia & $\begin{array}{l}\text { Detalles } \\
\text { fuera de } \\
\text { contexto }\end{array}$ \\
\hline
\end{tabular}

\section{Resultados}

Facultad de Ciencias de la Información - Universidad de La Laguna

Avenida César Manrique, s/n; Campus de Guajara

38071 La Laguna, Tenerife (Islas Canarias - España) 
Durante los seis meses de investigación, el diario El Espectador de Bogotá, publicó 42 $(44,52 \%)$ noticias relacionadas con el tema de la investigación; El Heraldo de Barranquilla, 19 (20,14\%); El Tiempo de Bogotá, 33 (34,98\%) y, El País de Cali, $12(12,72 \%)$, que trataran de manera directa o indirecta la cuestión estudiada.

El análisis se hizo teniendo en cuenta categorías como la variación de fechas, los protagonistas de las noticias, la agenda temática de los medios, la procedencia de la información, los detalles externos y el poder de las negrillas.

\subsection{Variación de fechas}

La variable de la fecha es de gran importancia al momento de conocer si el suceso tuvo un seguimiento constante por parte de los medios, pues el hecho de que se presente una continuidad en las noticias en vez de tan solo hacer mención el día del suceso, revela el buen tratamiento mediático del periódico mismo y por lo tanto se puede atacar una evidente desinformación del lector.

El cubrimiento informativo estudiado identificó varios días de alto impacto, destacándose, el discurso de Mahmoud Abbas, presidente de la ANP, ante la Asamblea General de la ONU, el discurso del presidente de Colombia, Juan Manuel Santos, ante la organización internacional y la llegada de Abbas a suelo colombiano para reunirse con el Gobierno como miembro temporal en el Consejo de Seguridad de las naciones unidas.

Durante los seis meses en que se presentaron las noticias alusivas a la propuesta de Palestina, se encontró repetición en las fechas según el carácter coyuntural de la información. De las 106 noticias estudiadas, 54 (50.9\%), se presentaron en septiembre, mes en el que se llevó a cabo la Asamblea General de las Naciones Unidas. Otras 40 (37.7\%) se publicaron en octubre, durante la visita de Mahmoud Abbas a Colombia. En cuanto a las otras fechas, 6 noticias (5.6\%) fueron publicadas en noviembre, 3 noticias (2.8\%) en julio, 2 $(1.8 \%)$ en agosto y $1(0.9 \%)$ en diciembre.

En este panorama, El Espectador publicó 42 noticias sobre el tema, 22 (52.3\%) de ellas en septiembre ( 5 de las cuales fueron el 23 de ese mes destacando el discurso del dirigente palestino ante la Asamblea General) y $17(40.4 \%)$ en octubre con Colombia como parte de los protagonistas, ya fuera por la visita del dirigente palestino o por la participación en situaciones relacionadas con el Consejo de Seguridad. Cabe destacar que solo quedaron 3 noticias publicadas en los meses faltantes, abarcando, principalmente, un contexto de los sucesos previos y posteriores a la Asamblea General.

El Heraldo de Barranquilla mostró un mayor interés en la visita de Abbas a Colombia en octubre y en las votaciones en el Consejo de Seguridad de la ONU en noviembre. En total, 
de las 19 noticias que publicó este periódico en su versión digital, 9 fueron en octubre $(47,3 \%), 7$ en septiembre $(36,8 \%)$ y $3(15,7 \%)$ en noviembre.

El diario El Tiempo mantuvo la tendencia, concentrando su información en los meses de septiembre y octubre, sobretodo en el cubrimiento de la cuestión durante la Asamblea de Naciones Unidas. En términos cuantitativos, de un total de 33 noticias, 19 (57.5\%) fueron publicadas en septiembre y $8(24.2 \%)$ en octubre, mientras que las restantes se presentaron en los otros meses: 2 en agosto y en julio (6.06\%), 1 en noviembre y 1 en diciembre (3.03\%).

Por último, El País de Cali presentó 12 notas y le dio mayor trascendencia a los sucesos durante fechas que representaban mayor importancia ala visita de Abbas a Colombia.

\subsection{Protagonistas de las noticias}

La categoría de los protagonistas de las noticias identifica a aquellos actores, personas u organizaciones, en los que se centra la información, ya sea por alguna declaración o decisión que hayan tomado, ya sea en el marco de la Asamblea General o en algún escenario previo o posterior a la cita.

Dentro de los protagonistas analizados estuvieron el Presidente de la Autoridad Nacional Palestina, Mahmoud Abbas, el Primer Ministro de Israel, Benjamin Netanyahu, el secretario general de la ONU, Ban Ki-moon y algunos delegados de las comitivas internacionales presentes en el evento. Por otro lado, entre las organizaciones más mencionadas en las noticias seleccionadas está la Organización de Naciones Unidas o subsidiarias de este organismo como la UNESCO.

Por el carácter nacional de la muestra, también se identificaron importantes personajes como Juan Manuel Santos y María Ángela Holguín, presidente y canciller de Colombia respectivamente, que además adquirieron mayor trascendencia por la participación temporal del país ante el Consejo de Seguridad, órgano decisorio en la toma de decisiones sobre el reconocimiento de nuevos Estados. Y precisamente este papel fue el que impulsaría al mandatario palestino Mahmoud Abbas a visitar Bogotá, entregándole protagonismo a la que entonces era la alcaldesa encargada de Bogotá, Clara López.

Abbas, quien fue el encargado de llevar la campaña pro Palestina y de asistir a la Asamblea de la ONU para leer la propuesta ante el pleno, fue el actor más resaltado en los diferentes informes, siendo el eje de 43 noticias (40,5\%), asociándose con la explicación de la propuesta de Palestina y su presentación en la Asamblea. De una menor manera, en relación con su visita a Colombia.

Luego está Juan Manuel Santos, presidente de Colombia, que en 25 notas tuvo el impacto necesario para ser el centro de la información $(23,5 \%$ de las noticias) y sentando, en la 
mayor parte de ellas, la posición de Colombia frente al delicado asunto coyuntural, defendiendo su alineamiento con los postulados presentados por Estados Unidos y Gran Bretaña.

Por su parte, el primer ministro de Israel, Benjamin Netanyahu se llevo el protagonismo en tan solo $8(7.5 \%)$ lo que demuestra que aunque tuvo presencia en momentos claves, como la Asamblea General, esta no tuvo más importancia que la que Mahmoud Abbas recibió de los medios de comunicación colombianos.

Posicionar el problema casi que exclusivamente en el hecho de que Palestina no es Estado, descuidando (sin afirmar que se desconozcan) las reclamaciones del lado israelí, crea un desbalance en la distribución de los protagonismos, impulsando a una lectura diferente del conflicto existente entre las dos naciones asiáticas.

Continuando con los protagonistas más relacionados en la información, se encuentran la canciller de Colombia María Ángela Holguín, en 6 ocasiones (5.6\%), la misma cantidad compartida por el presidente de Estados Unidos, Barack Obama.

Mientras tanto, organismos como la UNESCO tuvieron impacto en 3 noticias (2.8\%), que fueron aquellas ligadas a la aceptación que se dio a Palestina para pertenecer a la Organización. Ban Kim-Moon, secretario general de Naciones Unidas, fue otro personaje que a duras penas tuvo incidencia, apareciendo en 3 noticias (2.8\%), principalmente cuando cumplía con su papel como imagen de la ONU.

Por último, la ex alcaldesa encargada de Bogotá, Clara López, estuvo presente en 3 noticias (2.8\%) cuando Mahmoud Abbas visitó Colombia en el mes de octubre, resaltando su claro distanciamiento temático e ideológico con el presidente Juan Manuel Santos. Esto llamaría la atención ya que no es común que mandatarios locales o regionales opinen, oficialmente, sobre temas de la agenda internacional y mucho menos que contradigan la agenda nacional propuesta por el gobierno de turno.

\subsection{Marco temático de los medios}

Los medios de comunicación y sus periodistas toman decisiones editoriales que los llevan a presentar, de manera subjetiva, una visión particular de la realidad. Eligen qué noticias van a desvelar y cuáles no, habiendo seleccionado las fuentes que utilizarían para construir su información. Es esta selección, con todos sus filtros, la que al final llega a la opinión pública para que esta construya su propia visión de la realidad, en este caso, internacional.

Los diarios analizados construyeron su visión de esta realidad coyuntural sobre diez temas principales que fueron publicados diariamente, haciendo un seguimiento de cada uno de los 
sucesos ocurridos previamente a la Asamblea General y posterior al encuentro de las Naciones Unidas.

El primero de ellos se denominó Postura Palestina, que se refiere a la posición de que esta nación buscaba transmitir al mundo sobre la necesidad de ser reconocida como Estado, más de 60 años después de las declaraciones de 1947. Este eje temático fue, estadísticamente hablando, el más analizado por los medios, con el $23.6 \%$ de las noticias publicadas, siendo el periódico El Tiempo (12) el que más difusión le daría, y El Heraldo (2) el que menos.

Sobre esa misma línea se abrió la categoría de Apoyo a Palestina, al ver que la propuesta presentada a la Asamblea General recibió el apoyo de más de 115 países alrededor del mundo, entre los que se destacaron los 22 países de la Liga Árabe, y otros más cercanos a Colombia como Venezuela y Argentina. Esta información sería cubierta, de manera explícita, solamente por el periódico El Espectador con 2 noticias, el 1.9\% de la totalidad de notas analizadas, lo que mostraría la tendencia hacia donde se inclinaron los medios y su cubrimiento. Cabe aclarar, que se hicieron breves menciones al interior de otros artículos, pero solamente los reseñados le dedicarían espacio exclusivo.

La categoría de análisis Posición de Colombia reunió a los artículos que explicaron la posición de Colombia en la voz de la canciller María Ángela Holguín, que expuso las razones por las que el país no apoyaba la iniciativa y el presidente Juan Manuel Santos, que llamó a una negociación entre los israelitas y los palestinos para definir la propuesta. Esta fue presentada en el 15,1\%(16) de las noticias analizadas, siendo de nuevo El Espectador el diario que más difusión le daría.

En esta misma línea se abrió otra categoría de análisis denominada Exigencia de Diálogo, siguiendo el camino marcado por la petición de Colombia a Palestina de cambiar su postura e impulsarla a realizar un proceso de paz con Israel.

En cuanto al protagonismo de las organizaciones, se establecieron tres categorías de estudio, iniciando con Asamblea General de la ONU, en su reunión del 2011, que fue la protagonista de 23 noticias (21.7\%), siendo cubierta en una aplastante mayoría (15) por El Tiempo, pero reseñada por los cuatro diarios analizados. La UNESCO y su respaldo a Palestina, aceptándola como miembro, fue cubierta en 5 (4.7\%) notas, destacándose el interés de los dos diarios nacionales y la ausencia informativa en el cubrimiento de $\mathrm{El}$ Heraldo. Por último, las reuniones en el mes de noviembre del Consejo de Seguridad, en las que se discutió y se rechazó la propuesta palestina, tuvo un mayor interés en El Heraldo, que publicó 4 noticias, frente a 2 de El Tiempo y 1 de El Espectador para un $6.6 \%$ del total de las noticias analizadas.

La última categoría analizada fue la Gira de Mahmoud Abbas por Colombia que ocupó el $18.9 \%$ (20) de las noticias analizadas, siendo sobretodo cubierto por los diarios con base en Bogotá, que son los dos de cobertura nacional, en las que cada uno produjeron 7 noticias sobre el tema. 


\subsection{Procedencia de la información}

Frente a este punto, el análisis de las fuentes productoras de la información siempre abre el espacio a reflexiones sobre el trabajo periodístico en la aproximación a los temas publicados, especialmente si son de índole internacional.

Cuando se somete a las noticias a un análisis como el que se empleó en este estudio, este punto en particular permite ver que la variación en la selección y utilización de las diferentes fuentes, influye directamente en el marco que se crea de la información y su proyección sobre la opinión pública.

Se partió de la idea clara de que los medios, para temas internacionales, tienen pocas opciones para la producción de la información, ya que las obtienen de enviados especiales en el lugar de los hechos, que cubren las noticias de primera mano, o las extraen de las entregas de una agencia de noticias. Este último punto crea en si mismo otra problemática, y es que como la información producida es de carácter masivo, no es extraño que sobre ella los medios no ejecuten procesos de edición o simplemente la complementen, incluso con el trabajo de otras agencias, y terminen publicando varios diarios exactamente la misma noticia.

De acuerdo con el estudio, 50 noticias del total $(47.1 \%)$ son producto de las agencias; 22 noticias $(20.7 \%)$ solo dan el crédito de su página web; 9 noticias $(8.4 \%)$ provienen de corresponsales o de enviados especiales; 10 son producto de las redacciones de los medios $(9.4 \%) ;$ y 2 son de periódicos de otros países (1.8\%).

El Espectador de Bogotá publicó 17 noticias de agencias (40.4\%), 11 de ellas (26.1\%) con crédito de su página web (elespectador.com) haciendo uso de información de agencias internacionales, 3 de un corresponsal o enviado especial (7.1\%), dos noticias tomadas de un diario internacional (en ambos casos El País de España) y 7 noticias de su redacción internacional.

Por su parte, El Heraldo tomó 12 noticias de agencias (63,1\%); seis noticias $(31,5 \%)$ presentan el crédito de su página web y únicamente la información de una publicación $(5,2 \%)$ proviene de un enviado especial o corresponsal.

En cuanto a el diario El Tiempo, se evidencia que concentró la mayor parte de las noticias publicadas (13 que representa el $39.3 \%$ ) con el crédito de su página web (eltiempo.com) la mayoría de las veces citando cables de agencias. Luego, en 11 oportunidades destacó las agencias como procedencia de la información (33.3\%) y la agencia Efe fue mencionada la mayor parte de las veces.

Por último, 5 veces se publicaron notas de enviados especiales o corresponsales $(15,1 \%)$ más que todo en las noticias con información muy detallada sobre los acontecimientos que 
surgieron durante la Asamblea de la ONU en Nueva York, mientras la redacción del periódico tuvo 4 noticias (12,1\%) en asuntos de análisis; en este caso la redacción política fue la que desarrolló análisis específicamente sobre la posición de Colombia.

El País de Cali, con la menor participación en este tema coyuntural, tuvo como procedencia de la información únicamente agencias. Es el único medio de los cuatro estudiados que hace uso de los cables de la agencia de noticias AP (Associated Press), que ya deja ver la tendencia en el cubrimiento al ser esta una agencia de noticias norteamericana.

Sobre este camino, se pudo establecer que los medios utilizaron únicamente cuatro agencias: Reuters, AP, AFP y Efe. Según Rodríguez (2006), citado por Gelado (2009:250):

Por el carácter de las agencias y de sus clientes, las noticias corren el riesgo de concentrarse en ciertos aspectos que se consideran de interés esencial para el mundo desarrollado pero que no tienen tal carácter para gran parte del resto del mundo.

Gelado (2009: 270) también plantea quelas repercusiones de esta dependencia (agencias) son de gran importancia. Por un lado, la repetición de los mismos discursos en diferentes medios no hace sino disminuir el pluralismo, en tanto que hace que el número de publicaciones pase a ser irrelevante, ya que lo que importa al final es el número de agencias que surten de información a los medios.

Desde este punto se puede ver como la calidad noticiosa va en descenso buscando, así sea de forma repetitiva, llevar a los lectores un contexto informativo.

\subsection{El poder de la negrilla}

El lenguaje es la herramienta con la cual los seres humanos nos comunicamos. No sólo las palabras sino también los gestos y los sonidos conforman un punto esencial de la comunicación entre personas. Las palabras por sí solas tienen un gran poder y en especial si son utilizadas para presentar la postura de un tema en específico.

Los postulados del framing (Sádaba, 2011) afirman que todo ser humano enmarca la realidad que percibe dependiendo del lugar donde se encuentre, las cosas que haya vivido, su personalidad e inclusive su estrato social y cultura.

Una noticia es redactada por un periodista, su información es filtrada y ajustada a los parámetros y políticas de la empresa para la cual este está trabajando. Es por esto, que al serle asignado un espacio en específico, con una cantidad de caracteres exactos y poco tiempo para la redacción, el periodista debe hacer uso de sus mejores aptitudes lingüísticas ateniéndose siempre a la objetividad y la verdad. 
Algunos de estos elementos están en la categorización que obtienen como figura retórica y la función que desempeñan sobre el resto de palabras en una frase. No obstante en los textos sobresalen los detalles que resaltan las palabras y/o frases. La negrilla o negrita es una de esas figuras que influye en la interpretación del texto pues genera poder alrededor de la palabra misma.

La negrilla constituye un segundo filtro de la noticia pues actúa como una referencia de los detalles más importantes destacados dentro del texto ya redactado. El formato digital es el que más uso hace de esta herramienta ya que el lector no lee de forma completa un texto sino más bien lo escanea buscando detalles que atraigan su atención y lo informen en la menor cantidad de tiempo.

A lo largo de esta investigación ha sido posible percibir que cada diario tiene un comportamiento distinto en cuanto el uso del lenguaje y la presentación de los textos redactados. En cuanto a la morfología y en específico la figura negrilla, notamos que el diario El Espectador hace mayor uso de ella y El Heraldo el que menos.

Se encontraron 223 frases escritas en negrilla en las 106 noticias analizadas, en El Espectador se encontraron 197 en 42 notas, un promedio 4.7 por informe, en El País se escribieron 15 frases en 12 notas, promedio de 1.25, y en El Tiempo se publicaron 11 en 33 reportes, a un promedio de 0.33 .

Ahora, aunque en teoría las comillas buscan destacar la información que para los lectores puede ser más importante, también se encontraron ejemplos en los que el recurso no fue bien utilizado, como en la noticia de El Espectador "Petición de Palestina de ingreso a la ONU pasó a comité de nuevas adhesiones" del 28 de septiembre de 2012, en la que se utilizaron en 841 palabras un total de 18 veces la negrilla, generando confusión e inutilizando la posible efectividad del recurso.

En El Tiempo la figura tan solo se utiliza cuando la información realmente lo amerita o cuando la noticia como tal es muy larga, mientras que en El País de Cali se utilizó lo suficiente para destacar un punto importante sin opacar el resto de la información ni omitir la ayuda.

Por último, está el periódico barranquillero El Heraldo que optó por no utilizar este recurso morfológico lo que puede tornarse un elemento de doble filo teniendo en cuenta que en la comunicación digital el lenguaje es diferente a aquel de la prensa escrita e impresa.

Del análisis cualitativo realizado en esta investigación se encontró que el uso de la figura morfológica negrilla funciona, en primera instancia, como un apoyo a los titulares e intertítulos de la noticia, de modo que si el lector opta por dirigirse únicamente a estos tendrá un conocimiento básico sobre los hechos. Aunque es evidente que el uso de esta figura resume 
aún más las noticias haciendo menos importante la lectura total del documento publicado, es indispensable en el mundo globalizado pues como el público promedio de los periódicos digitales es aquel que escanea el texto y no el que lo digiere con tanta atención como el que lee el diario impreso.

De un total de 106 noticias publicadas en los cuatro periódicos nacionales de mayor tiraje a nivel nacional en Colombia en su versión digital se encontraron que el 33\% de las noticias publicadas utilizando la negrilla como un complemento a los titulares de la nota misma.

En su gran mayoría -15 de 28- las frases en negrilla pueden ser vistas como herramientas de apoyo; de las 13 restantes 12 son citas textuales, con comillas, y una cita parafraseada. Esta última, tomada de la noticia "Clara López entregó llaves de la ciudad al presidente palestino" publicada el 10 de octubre de 2011 en el diario El Espectador, dice lo siguiente: "entregarle las llaves de la ciudad es un acto de amistad y solidaridad con el pueblo palestino". Al no encontrarse con un entrecomillado dentro del texto del periódico parecería que es una afirmación del autor pues no ponerle en negrilla la parte en la que se esclarece su procedencia puede confundir al lector y por ende, la percepción misma de la noticia.

Como bien se planteó previamente, la figura morfológica tiene como misión destacar un punto sobre el resto del texto por lo que es indispensable el tener sumo cuidado en el momento de seleccionar cuales son las frases que van a ser escogidas para señalar con destacables pues se debe verificar que esclarezcan e informen en vez de confundir y desinformar.

\section{Conclusiones}

De acuerdo a un estudio minucioso de los resultados expuestos previamente, se puede concluir que el tratamiento informativo que se le dio a la información sobre la propuesta de adhesión como Estado por parte de Palestina, ante la Organización de Naciones Unidas, fue sumamente pobre por varios motivos.

En primera instancia, la mayoría de noticias fueron redactadas con la información provista por los cables de las agencias EFE, Reuters y AFP, con un total del $41 \%$, más otro $30 \%$ que le da crédito a su página web, pero soportando su información en las mismas agencias.

La mayor cantidad de noticias fueron publicadas en septiembre y octubre de 2011, lo cual tiene sentido si tenemos en cuenta que en septiembre fue la Asamblea General de la ONU y en octubre la visita del presidente palestino, Mahmoud Abbas, a Colombia y la resolución del Consejo de Seguridad sobre el tema palestino.

En cuanto a esto último se hizo un corto registro sobre las conclusiones del organismo frente a la propuesta de Palestina por lo que se podría afirmar que la última parte de la cobertura fue nula en comparación con los momentos clave. 
También es indispensable destacar que en agosto y noviembre hubo gran movimiento coyuntural por las reuniones previas a la Asamblea General y la campaña palestina en torno a las mismas sin mucha cobertura. Se notó que la totalidad de lectores de estos cuatro diarios debieron informarse de la situación con muy poco material noticioso que además provenía de la misma fuente en su mayoría la agencia EFE, por lo que la información no sólo venía previamente encuadrada, sino que además era casi, por no decir la misma, en todos los periódicos estudiados.

Con respecto a las agencias es crucial tener en cuenta que la mayoría de sus fuentes se remiten a la información de los diarios locales palestinos e israelíes que de por sí han encuadrado la noticia según el lado de la frontera en el cual se encuentran y el cómo los afecta el conflicto que viven en ese momento. Es decir que si la publicación proviene de Palestina, la cobertura dada a Mahmoud Abbas y su campaña por una silla en la ONU tendrá un mayor despliegue mediático que aquel que tendría en los diarios israelíes.

También se encontraron que los protagonistas de cada noticia fueron en principio las autoridades tanto de las naciones participantes como de la Organización de las Naciones Unidas y la UNESCO. En un total 106 noticias tan solo tres fueron relacionadas con el conflicto directo de los pueblos y con sus manifestaciones y posturas con respecto a la posición de Israel y Palestina frente a la ONU y el mundo, y viceversa. Estas tres noticias fueron " 5 palestinos recorrerán los $1.800 \mathrm{~km}$ en cicla por reconocimiento de la ONU" publicada en el diario El Espectador, "En un mes palestinos pedirán adhesión a la ONU" del diario El Tiempo y "Fiesta palestina en Cisjordania, marchas en Israel" del diario El Tiempo.

Además, es indispensable recalcar que los medios de comunicación estudiados optaron por tratar con suma importancia temas cuya relevancia se salía del tema base de la propuesta de Palestina. Un ejemplo de esto fue la entrega de llaves a Mahmoud Abbas por parte de la alcaldesa encargada de Bogotá, Clara López, y el recorrido que el presidente Palestino haría en la capital de nuestro país. A diferencia de los hechos primordiales que debieron ser tratados con mayor énfasis, la visita del presidente de la ANP, en cuanto en cuanto al horario y lugares de visita que haría cada lugar de Bogotá, los primeros tuvieron un cubrimiento pobre.

Al ofrecer una sección de noticias internacionales, el diario está ofreciendo a los lectores un lugar donde pueden estar informados de los sucesos mundiales de mayor importancia. Sin embargo, la información pierde calidad y objetividad cuanto se encuentra el mismo titular y los mismos contenidos en mapas de un periódico. Así mismo, el estudio que se realizó demostró que las fuentes primarias se mantienen como únicas por lo que la profundización es muy poca en las publicaciones. 
Las posturas de El Espectador y El Tiempo se mostraron muy diferentes, teniendo en cuenta dos puntos clave como la Asamblea General y la visita de Abbas a Colombia. El Espectador tuvo la mayor cobertura en los dos meses clave (septiembre y octubre).

Teniendo en cuenta estos datos, se puede afirmar que El Tiempo y El Espectador fueron los diarios que más notas aportaron sobre este proceso que tuvo Palestina, mientras que El Heraldo y El País tuvieron una menor participación.

Finalmente, es posible denotar que las hipótesis planteadas en este ejercicio se cumplieron a cabalidad en cuanto que el cubrimiento de noticias internacionales por parte de los medios de comunicación escritos nacionales no es solo sumamente pobre sino poco sustentando y corroborado, o como se planteó en la hipótesis de este ejercicio, la información no es muy amplia ni en cantidad ni en calidad.

Y a pesar de que estos medios son considerados como los más importantes de Colombia, no llenaron las expectativas informativas a pesar de pertenecer a grupos económicos que trascienden las barreras geopolíticas actuales. Así mismo, se puede deducir que como Colombia no apoyó a Palestina (al abstenerse de votar), los medios tampoco le dieron importancia alguna a la no participación de nuestro país en la adhesión de esta nación de Oriente Medio como un Estado independiente de Israel.

\section{Bibliografía}

Ahmed, M. (2009): The Israeli-Palestinian Conflict in American, Arab, and Britich Media: CorpusBased Critical Discourse Analysis. Applied Linguistics and English as a Second Language Dissertations. $\quad$ Paper 12.2 Disponible en internet: http://digitalarchive.gsu.edu/cgi/viewcontent.cgi?article=1012\&context=alesl diss Extraído el 20 de abril de 2012.

Amadeo, B. (2002): La teoría del framing: Los medios de comunicación y la transmisión de significados en Revista de Comunicación, Perú. Vol. 1, 6-32.

Arts, B.; Noortmann, M. \& Reinalda, B. (2001): Non-state actors in international relations. Aldershot: Ashgate.

Aruguete, N. (2010): Los encuadres noticiosos en los medios argentinos. América Latina Hoy, Vol. 54, 113-137.

AS - Asamblea General de la ONU (1993): Carta de fecha 8 de octubre de 1993 dirigida al Secretario General por los Representantes Permanentes de los Estados Unidos de América y la Federación de Rusia ante las Naciones Unidas. Disponible en la URL: http://www.palestina.int.ar/files/Oslo.pdf Revisado el 14 de mayo de 2012.

Bacalor, G. (2011): Los medios son cómplices del conflicto árabe israelí. Disponible en internet: http://www.aurora-israel.co.il/articulos/israel/Titular/38597/ Extraído el 18 de mayo de 2012. 
Barreñada, I. (2011): La iniciativa palestina en Naciones Unidas: expectativas posibilidades y riesgos. Real Instituto Elcano. España. Disponible en la URL: http://ribei.org/2194/1/ARI-132-2011.pdf. Extraído el 17 de abril de 2012.

Berganza C., M. R. (2008): Medios de comunicación, espiral del cinismo y desconfianza política. ZER, 13, 25, 121-139.

Berger, B.K. \& Reber, B.H. (2005): "Framing analysis of activist rhetoric: How the Sierra Club succeeds or fails at creating salient messages" en Public Relations Review, Vol. 31, Issue 2, pp. 185-195.

Berger, P. L. \& Luckmann, T. (2001): La construcción social de la realidad, 17르 reimp. Buenos Aires: Amorrortu Editores.

Bosemberg, L. (2009): El conflicto Palestino-Israelí, una propuesta para la negociación. Colombia internacional $\quad N^{\circ} 69$ pp 142-161. Disponible en internet: http://www.auroraisrael.co.il/articulos/israel/Titular/38597/ Extraído el 18 de mayo de 2012.

Brewer, P.R., Graf, J. \& Willnat, L. (2003): Priming of Framing. Gazette The International Journal for Communication Studies, Vol. 65, 2, 493-508.

Broullón, G.; Hernández, T.; López, X.; Pereira, J. (2005): Los observatorios de comunicación. Revista latinoamericana de comunicación Chasqui. No 90. Pp 38 - 45. Disponible en internet: http://redalyc.uaemex.mx/src/inicio/ArtPdfRed.jsp?iCve=16009008. Extraído el 3 de junio de 2012.

Brunetto, M. J. (2011). El proceso de creación del Estado de Israel: ¿origen político de un conflicto sin fin en la región del Cercano Oriente? Monografía de profesorado. Centro de estudios de Relaciones Internacionales. Disponible en URL: http://cerrii.org/descargas/hrrii1 proceso creacion estado israel.pdf. Revisado el 25 de enero de 2012.

Caruana, L. (2002): La Crisis de Oriente Medio: Palestina. Jean Monnet European centre of Excellence. Disponible en Internet: http://www.idee.ceu.es/Portals/O/Publicaciones/La-crisis-deOriente-Medio-Palestina.pdf. Revisado el 18 de mayo de 2012.

Centro de noticias de la ONU (2011) Los mayores obstáculos para Estado palestino son políticos, afirma ONU. Disponible en la URL: http://www.un.org/spanish/News/fullstorynews.asp?NewsID=21884. Revisado el 22 de febrero de 2012.

Chapman P., S. (2004): A qualitative framing analysis of the NAACP's public relations response to the 2000 presidential election. Public Relations Review, 31, 63-71.

Cohen, B. (1963): The Press and the Foreign Policy. Princenton University Press, Princenton.

Córdoba, A. M. (2009): La línea editorial de ABC, El País, El Mundo y La Vanguardia frente al conflicto palestino-israelí: 1993-2004. Aurel Cárdona Jaume. Tesis doctoral. Universidad de Navarra, Pamplona. 
Córdoba, A. M. (2011): El conflicto Palestino-Israelí visto desde España: oscilaciones y tendencias de la opinión pública. Ámbitos №20. Disponible en internet: http://grupo.us.es/grehcco/ambitos20/08cordoba.pdf Extraído el 18 de mayo de 2012.

Criscaut, A. (2008): El Nacionalismo palestino frente al Estado de Israel. El Sufrimiento como Identidad. Centro de estudios del Medio Oriente contemporáeo. Argentina. Dispinible en internet: http://www.cemoc.com.ar/Documento\%20de\%20Trabajo\%2002-2008.pdf. Extraído el 25 de mayo de 2012.

D'angelo, P. (2002): "News framing as a multi-paradigmatic research program: A response to Entman" en Journal of Communication Vol. 52, № 4, pp. 870-888.

Daher, S. (2002): Diagnóstico de Palestina. Relaciones Internacionales. Número 22. Disponible en internet: http://www.iri.edu.ar/revistas/revista dvd/revistas/R22/ri\%2022\%20estudio\%20akel.pdf. Extraído el 22 de abril de 2012.

De Currea Lugo, V. (2005): Palestina: entre la trampa del muro y el fracaso del derecho. Icaria editorial.

Díaz, J.; Fertonani, A. (s.t.). La participación de Palestina y la creación del estado de Israel en la prensa gráfica de Córdoba en 1948. Los casos de la voz del interior y los principios. Disponible en la URL:_http://www.cea.unc.edu.ar/africaorientemedio/contrapdfs/0506/6\%20Diaz\%20Fertonani.pdf. Revisado el 24 de enero de 2012.

DIPNU (Departamento de Información Pública de las Naciones Unidas) (2003). La Cuestión palestina y las Naciones Unidas. Disponible en internet: http://www.un.org/spanish/Depts/dpi/palestine/. Revisado el 24 de mayo de 2012.

Entman, R. (2004): Projects of Power: Framing News, Public Opinion, and U.S. Foreign Policy. Chicago: Univ. Chicago Press.

Entman, R. (2006): Framming: toward clarification of a fractured paradigm en Journal of Communication. Oxford, Blackwell Publishing. Vol. 43, №4. Pp 51-58.

García, D. (2000): Globalización, nuevas tecnologías y tratamiento periodístico de la información en Revista Latina de comunicación social, № 27, pp. 1-17.

Gelado, R. (2009): La dependencia de la prensa española hacia las agencias de noticias. COMUNICACIÓN Y SOCIEDAD. Vol. XXII. Número 2.

Giddens, A. (1990): The Consequences of Modernity. Stanford: Stanford University Press. ${ }^{*}$ Citado

Gimenez A., P. (2006): Una nueva visión del proceso comunicativo: La teoría del enfoque. Revista Comunicación y Hombre, 2, 55-66.

Gitlin, T. (1980): The whole world is watching: mass media in the making \& unmaking of the New Left. Berkeley: University of California Press.

Goffman, E. (1974): Frame Analysis: An Essay on the Organization of Experience. New York. 
Gómez Mendoza, M. A. (1999): "Análisis de contenido cualitativo y cuantitativo. Definición, clasificación y metodología" en Revista de ciencias humanas, $N^{\circ} 20$ (en línea) http://www.utp.edu.co/ chumanas/revistas/revistas/rev20/gomez.htm [Consulta: 4 de julio de 2011].

Hallahan, K. (1999): Seven Models of Framing: Implications for Public Relations. Journal of Public Relations Research, 11, 3, 205-242.

Held, D. (2007): "Reframing global governance: Apocalypse son or reform!" en HELD, D. \& McGREW, A. (eds.) (2007): Globalization Theory: Approaches and Controversies. Cambridge: Polity Press.

Husseini, H. (2011): Palestina y la ONU: el camino legítimo para un Estado. Política exterior Vol. 25 No. 143. Disponible en internet: http://www.politicaexterior.com/articulo?id=4705. Extraído el 25 de enero de 2012.

lyengar, S. (1991): Is anyone responsible? : how television frames political issues. Chicago: University of Chicago Press.

Kaplan, Nora (2009). La construcción discursiva del evento conflictivo en las noticias por televisión. U. Central de Venezuela. Disponible en internet: http://www.dissoc.org/recursos/tesis/Tesis\%20Nora\%20Kaplan.pdf Extraído el 18 de mayo de 2012.

Machover, M. (2009): Israelíes y palestinos: Conflicto y solución. International Socialist Review. Disponible en internet: http://www.isreview.org:80/issues/65/feat-machover.shtml. Extraído el 18 de mayo de 2012.

Martínez Carreras, J. (1999). 50 años del Estado de Israel. Cuadernos de historia contemporánea. Número 21. Pg 355-363. Disponible en internet: http://dialnet.unirioja.es/servlet/dcart?info=link\&codigo=905831\&orden=1

Naciones Unidas (2003). La cuestión de Palestina y las Naciones Unidas. Disponible en internet: http://www.un.org/spanish/Depts/dpi/palestine/. Extraído el 14 de mayo de 2012.

Naciones Unidas (2012). Cuestión de Palestina. Disponible en internet: http://www.cinu.org.mx/temas/palestina.htm\#orig. Extraído el 22 de abril de 2012.

Nye, J. (2004): Soft power: The Means to Success In World Politics. New York: Public Affairs.

Portilla Gómez, J. M. (2006). El derecho internacional ante la ocupación de los territorios palestinos y el muro de separación. Anuario mexicano del derecho. Vol. VI. Disponible en internet: http://revistas.unam.mx/index.php/amdi/article/view/16616 Revisado el 25 de enero de 2012.

Rahal, D. (2001). El colapso del proceso de paz palestino-israelí. México. Disponible en internet: http://redalyc.uaemex.mx/redalyc/pdf/267/26701508.pdf. Extraído el 24 de abril de 2012.

Ramírez León, J. L. (1989) La Proclamación del Estado Palestino y sus Implicaciones en las relaciones Internacionales de Colombia. Colombia Internacional, Universidad de los Andes. Enero 
marzo, páginas 3-10. Disponible en la URL: http://colombiainternacional.uniandes.edu.co/view.php/36/buscar.php?keyword=pol\%C3\%ADtica+e xterior+colombia\&search=Buscar Revisado el 21 de enero de 2012.

Ramírez, Marcelo (2011). Crisis en el mundo árabe. Análisis desde el 'paradigma de la transición'. Revista Enfoques. Vol. IX. №14. Santiago. Disponible en internet: http://www.politicaygobierno.cl/wp-content/uploads/2011/07/Enf14 Ramirez.pdf. Extraido de 19 de mayo de 2012.

Reese, S. D.; Gandy, O. H. Y Grant, A. E. (2001): Framing public life: Perspectives on media and our understanding of the social world. Mahwah, NJ: Lawrence Erlbaum Associates.

Sádaba, M.T. (2001). Origen, aplicación y límites de la "teoría de encuadre" (framing) en comunicación. Comunicación y Sociedad, vol. XIV, n. 2, pp. 143-175. Disponible en internet: http://www.unav.es/fcom/comunicacionysociedad/es/articulo.php?art id=335. Extraído el 12 de marzo de 2012.

Sádaba, M.T. (2004): Enfoques periodísticos y marcos de participación política. Una aproximación conjunta a la teoría del encuadre. Política y Sociedad. http://revistas.ucm.es/cps/11308001/articulos/POSO0404130065A.PDF [Consulta: 30 de abril de 2010]

Sádaba, M.T. (2006). Framing, una teoría para los medios de comunicación. Pamplona, Ulzama Digital.

Sádaba, M.T. (2008): Framing: el encuadre de las noticias. El binomio terrorismo-medios, La Crujía, Buenos Aires.

Sádaba, M.T. \& Rodríguez, J. (2007): "La construcción de la agenda de los medios. El debate del estatut en la prensa española”, en Ámbitos, $n^{\circ}$. 16, pp. 187-211.

Scheufele, D. (1999): Framing as a Theory of Media Effects. Journal of Communication, 103-121

Scheufele, D. A. (2000): "Agenda-setting, priming and framing revisited: another look at cognitive effects of political communication" en Mass Communication and Society, Vol. 3, № 2-3, pp. 297316.

Soderblom, J. (2003). A State of Inequity: The UN Partition Plan of 1947. The Terrorism Intelligence Centre, Canberra. Disponible en internet: http://world-ice.com/Articles/Inequity.pdf. Extraído el 21 de mayo de 2012.

Sollich, R. (2011). Estado Palestino: ¿un riesgo de seguridad? Deutsche Welle. Seeptiembre 23 de 2011. Disponible en la URL: http://www.dw.de/dw/article/0,15412376,00.html. Revisado el 24 de febrero de 2012.

Spizzamiglio, C.A. (2005). El Consejo de Seguridad en el tratamiento del conflicto entre palestinos e israelíes: 1947-2005. Disponible en internet: http://www.saap.org.ar/esp/docs- 
congresos/congresossaap/VII/programa/paneles/e/e1/spizzamiglio.pdf. Extraído el 22 de enero de 2012.

Tankard, J.W., et al (1991): Media Frames: Approaches to Conceptualization and Measurement. Paper presented at the Association for Education in Journalism and Mass Communication, Boston, Agosto.

Tewksbury, D. Y Scheufele, D.A, (Eds.) (2007): "Special Issue on Framing, Agenda Setting, \& Priming: Agendas for Theory and Research" en Journal of Communication, Vol. 57, № 1, pp. 9173.

Tuchman, G. (1978): Making News. A study in the Construction of Reality. Nueva York, The Free Press

Tuchman, G. (1983): La producción de la noticia: estudio sobre la construcción de la realidad. México: Gustavo Gili.

Tuchman, Gaye (2010). Making news by doing work. The American Journal of Sociology, Vol. 79, No. 1 (Jul., 1973), pp. 110-13 The University of Chicago Press. Disponible en internet: http://es.scribd.com/doc/62770599/Tuchman-News-Rituals. Extraído el 21 de abril de 2012.

Tuñez, M. Y Guevara, M. (2009): Framing por proximidad como criterio de noticiabilidad: la curva de las ausencias. Revista Latina de Comunicación Social, 12, 64, 1030-1044.

UNESCO (1978). Declaración de la UNESCO en 1978 sobre los principios fundamentales relativos a la contribución de los medios de comunicación al refuerzo de la paz y la comprensión internacional, a la promoción de los derechos humanos y a la lucha contra el racismo, el apartheid y la incitación a la guerra. Disponible en internet: http://unesdoc.unesco.org/images/0018/001836/183664so.pdf Extraído el 23 de marzo de 2012.

UNESCO (1983). Código de ética: UNESCO, Principios internacionales de ética profesional en periodismo. París. Unesco.

Urrutia, Pamela (2011). Conflicto palestino-israelí ¿Más proceso que paz? QUADERNS DE CONSTRUCCIÓ DE PAU № $23 . \quad$ Disponible en internet: http://ddd.uab.cat/pub/quaconpau/quaconpau a2011m9n23.pdf. Extraído el 24 de abril de 2012.

Vicente, M. Y López, P (2009): "Resultados actuales de la investigación sobre framing: sólido avance internacional y arranque de la especialidad en España" en Revista ZER, Vol. 14, № 26, pp. 13-34.

Vreese, C.H. (2004): The Effect of Frames in Political Television News on Issue Interpretation and salience. J\&MC Quartely, Vol. 81, 2, 36-52.

Vreese, C.H. (2005): News framing: theory and typology. Information Design Journal + Document Design, 13, 1, 51-62 


\section{Forma de citar este artículo en bibliografías}

VELÁSQUEZ ESPINOSA, J. D. (2012): "El marco informativo de la iniciativa estatal de palestina en los medios colombianos", en Revista PANGEA, 3, páginas 91 a 117. Red Académica Iberoamericana de Comunicación. Recuperado el de de 2 de: http://www.revistapangea.org 